MOL CAS2 SPR1 SPI CAR1 CAR2 CER FAM PLA MIN1 MOCK Fv1" Fv1 ${ }^{\text {b }}$ 


\section{Figure S2}

Western blot analysis of Mus dunni cells transduced with Fv1s from wild mice. MDTF cells were transduced with retroviral vectors carrying the Fv1 gene from wild mice and the EYFP marker such that $50 \%$ of the cells were transduced, as determined by flow cytometry. A western blot analysis was performed 3 days post transduction using $25 \mu \mathrm{g}$ of total protein from the cell extracts as previously described (48). 